IJMMS 31:1 (2002) 37-42

PII. S0161171202110386

http://ijmms.hindawi.com

(c) Hindawi Publishing Corp.

\title{
THE GALOIS ALGEBRAS AND THE AZUMAYA GALOIS EXTENSIONS
}

\author{
GEORGE SZETO and LIANYONG XUE
}

Received 26 October 2001

Let $B$ be a Galois algebra over a commutative ring $R$ with Galois group $G, C$ the center of $B, K=\{g \in G \mid g(c)=c$ for all $c \in C\}, J_{g}=\{b \in B \mid b x=g(x) b$ for all $x \in B\}$ for each $g \in K$, and $B_{K}=\left(\oplus \sum_{g \in K} J_{g}\right)$. Then $B_{K}$ is a central weakly Galois algebra with Galois group induced by $K$. Moreover, an Azumaya Galois extension $B$ with Galois group $K$ is characterized by using $B_{K}$.

2000 Mathematics Subject Classification: 16S35, 16W20.

1. Introduction. Let $B$ be a Galois algebra over a commutative ring $R$ with Galois group $G$ and $C$ the center of $B$. The class of Galois algebras has been investigated by DeMeyer [2], Kanzaki [6], Harada [4, 5], and the authors [7]. In [2], it was shown that if $R$ contains no idempotents but 0 and 1 , then $B$ is a central Galois algebra with Galois group $K$ and $C$ is a commutative Galois algebra with Galois group $G / K$ where $K=\{g \in G \mid g(c)=c$ for all $c \in C\}$ [2, Theorem 1]. This fact was extended to the Galois algebra $B$ over $R$ containing more than two idempotents [6, Proposition 3], and generalized to any Galois algebra $B$ [7, Theorem 3.8] by using the Boolean algebra $B_{a}$ generated by $\left\{0, e_{g} \mid g \in G\right.$ for a central idempotent $\left.e_{g}\right\}$ where $B J_{g}=B e_{g}$ and $J_{g}=\{b \in B \mid b x=g(x) b$ for all $x \in B\}$ for each $g \in G$ [6]. The purpose of this paper is to show that there exists a subalgebra $B_{K}$ of $B$ such that $B_{K}$ is a central weakly Galois algebra with Galois group $\left.K\right|_{B_{K}}$ induced by $K$ where a weakly Galois algebra was defined in [8] and that $B_{K} B^{K}$ is an Azumaya weakly Galois extension with Galois group $\left.K\right|_{B_{K} B^{K}}$ where an Azumaya Galois extension was studied in [1]. Thus some characterizations of an Azumaya Galois extension $B$ of $B^{K}$ with Galois group $K$ are obtained, and the results as given in $[2,6]$ are generalized.

2. Definitions and notations. Throughout, let $B$ be a Galois algebra over a commutative ring $R$ with Galois group $G, C$ the center of $B$, and $K=\{g \in G \mid g(c)=$ $c$ for all $c \in C$ \}. We keep the definitions of a Galois extension, a Galois algebra, a central Galois algebra, a separable extension, and an Azumaya algebra as defined in [7]. An Azumaya Galois extension $A$ with Galois group $G$ is a Galois extension $A$ of $A^{G}$ which is a $C^{G}$-Azumaya algebra where $C$ the center of $A$ [1]. A weakly Galois extension $A$ with Galois group $G$ is a finitely generated projective left module $A$ over $A^{G}$ such that $A_{l} G \cong \operatorname{Hom}_{A} G(A, A)$ where $A_{l}=\left\{a_{l}\right.$, a left multiplication map by $\left.a \in A\right\}$ [8]. We call that $A$ is a weakly Galois algebra with Galois group $G$ if $A$ is a weakly Galois extension with Galois group $G$ such that $A^{G}$ is contained in the center of $A$ and that 
$A$ is a central weakly Galois algebra with Galois group $G$ if $A$ is a weakly Galois extension with Galois group $G$ such that $A^{G}$ is the center of $A$. An Azumaya weakly Galois extension $A$ with Galois group $G$ is a weakly Galois extension $A$ of $A^{G}$ which is a $C^{G}$-Azumaya algebra where $C$ the center of $A$.

3. A weakly Galois algebra. In this section, let $B$ be a Galois algebra over $R$ with Galois group $G, C$ the center of $B, B^{G}=\{b \in B \mid g(b)=b$ for all $g \in G\}$, and $K=\{g \in$ $G \mid g(c)=c$ for all $c \in C\}$. Then, $B=\oplus \sum_{g \in G} J_{g}=\left(\oplus \sum_{g \in K} J_{g}\right) \oplus\left(\oplus \sum_{g \notin K} J_{g}\right)$ where $J_{g}=\{b \in B \mid b x=g(x) b$ for all $x \in B\}$ [6, Theorem 1]. We denote $\oplus \sum_{g \in K} J_{g}$ by $B_{K}$ and the center of $B_{K}$ by $Z$. Clearly, $K$ is a normal subgroup of $G$. We show that $B_{K}$ is an Azumaya algebra over $Z$ and a central weakly Galois algebra with Galois group $\left.K\right|_{B_{K}}$.

THEOREM 3.1. The algebra $B_{K}$ is an Azumaya algebra over $Z$.

Proof. By the definition of $B_{K}, B_{K}=\oplus \sum_{g \in K} J_{g}$, so $C\left(=J_{1}\right) \subset B_{K}$. Since $B$ is a Galois algebra with Galois group $G$ and $K=\{g \in G \mid g(c)=c$ for all $c \in C\}$, the order of $K$ is a unit in $C$ by [6, Proposition 5]. Moreover, $K$ is an $C$-automorphism group of $B$, so $B_{K}$ is a $C$-separable algebra by [5, Proposition 5]. Thus $B_{K}$ is an Azumaya algebra over $Z$.

In order to show that $B_{K}$ is a central weakly Galois algebra with Galois group $\left.K\right|_{B_{K}}$, we need two lemmas.

LEMMA 3.2. Let $L=\left\{g \in K \mid g(a)=a\right.$ for all $\left.a \in B_{K}\right\}$. Then, $L$ is a normal subgroup of $K$ such that $\bar{K}(=K / L)$ is an automorphism group of $B_{K}$ induced by $K$ (i.e., $\left.K\right|_{B_{K}} \cong \bar{K}$ ).

Proof. Clearly, $L$ is a normal subgroup of $K$, so for any $h \in K$,

$$
h\left(B_{K}\right)=\oplus \sum_{g \in K} h\left(J_{g}\right)=\oplus \sum_{g \in K} J_{h g h^{-1}}=\oplus \sum_{g \in h K h^{-1}} J_{g}=\oplus \sum_{g \in K} J_{g}=B_{K}
$$

Thus $\left.K\right|_{B_{K}} \cong \bar{K}$.

LEMMA 3.3. The fixed ring of $B_{K}$ under $K,\left(B_{K}\right)^{K}=Z$.

PRoof. Let $x$ be any element in $\left(B_{K}\right)^{K}$ and $b$ any element in $B_{K}$. Then $b=\sum_{g \in K} b_{g}$ where $b_{g} \in J_{g}$ for each $g \in K$. Hence $b x=\sum_{g \in K} b_{g} x=\sum_{g \in K} g(x) b_{g}=\sum_{g \in K} x b_{g}=$ $x \sum_{g \in K} b_{g}=x b$. Therefore $x \in Z$. Thus $\left(B_{K}\right)^{K} \subset Z$. Conversely, for any $z \in Z$ and $g \in K$, we have that $z x=x z=g(z) x$ for any $x \in J_{g}$, so $(g(z)-z) x=0$ for any $x \in J_{g}$. Hence $(g(z)-z) J_{g}=\{0\}$. Noting that $B J_{g}=J_{g} B=B$, we have that $(g(z)-z) B=\{0\}$, so $g(z)=z$ for any $z \in Z$ and $g \in K$. Thus $Z \subset\left(B_{K}\right)^{K}$. Therefore $\left(B_{K}\right)^{K}=Z$.

THEOREM 3.4. The algebra $B_{K}$ is a central weakly Galois algebra with Galois group $\left.K\right|_{B_{K}} \cong \bar{K}$.

Proof. By Lemma 3.3, it suffices to show that (1) $B_{K}$ is a finitely generated projective module over $Z$, and (2) $\left(B_{K}\right)_{l} \bar{K} \cong \operatorname{Hom}_{Z}\left(B_{K}, B_{K}\right)$. Part (1) is a consequence of Theorem 3.1. For part (2), since $B_{K}$ is an Azumaya algebra over $Z$ by Theorem 3.1 again, $B_{K} \otimes_{Z} B_{K}^{o} \cong \operatorname{Hom}_{Z}\left(B_{K}, B_{K}\right)$ [3, Theorem 3.4, page 52] by extending the map $(a \otimes b)(x)=a x b$ linearly for $a \otimes b \in B_{K} \otimes_{Z} B_{K}^{o}$ and each $x \in B_{K}$ where $B_{K}^{o}$ is the 
opposite algebra of $B_{K}$. By denoting the left multiplication map with $a \in B_{K}$ by $a_{l}$ and the right multiplication map with $b \in B_{K}$ by $b_{r},(a \otimes b)(x)=\left(a_{l} b_{r}\right)(x)=a x b$. Since $B_{K}=\oplus \sum_{g \in K} J_{g}, B_{K} \otimes_{Z} B_{K}^{o}=\sum_{g \in K}\left(B_{K}\right)_{l}\left(J_{g}\right)_{r}$. Observing that $\left(J_{g}\right)_{r}=\left(J_{g}\right)_{l} \bar{g}^{-1}$ where $\overline{\mathfrak{g}}=\left.\left.g\right|_{B_{K}} \in K\right|_{B_{K}} \cong \bar{K}$, we have that $B_{K} \otimes_{Z} B_{K}^{o}=\sum_{g \in K}\left(B_{K}\right)_{l}\left(J_{g}\right)_{r}=\sum_{g \in K}\left(B_{K}\right)_{l}\left(J_{g}\right)_{l} \overline{\mathfrak{g}}^{-1}=$ $\sum_{g \in K}\left(B_{K} J_{g}\right)_{l} \bar{g}^{-1}$. Moreover, since $B J_{g}=B$ for each $g \in K$ and $B=\oplus \sum_{h \in G} J_{h}=B_{K} \oplus$ $\left(\oplus \sum_{h \notin K} J_{h}\right), B_{K} \oplus\left(\oplus \sum_{h \notin K} J_{h}\right)=B=B J_{g}=B_{K} J_{g} \oplus\left(\oplus \sum_{h \notin K} J_{h} J_{g}\right)$ such that $B_{K} J_{g} \subset B_{K}$ and $\oplus \sum_{h \notin K} J_{h} J_{g} \subset \oplus \sum_{h \notin K} J_{h}$. Hence $B_{K} J_{g}=B_{K}$ for each $g \in K$. Therefore $B_{K} \otimes_{Z} B_{K}^{o}=$ $\sum_{g \in K}\left(B_{K} J_{g}\right)_{l} \overline{\mathfrak{g}}^{-1}=\sum_{g \in K}\left(B_{K}\right)_{l} \overline{\mathfrak{g}}^{-1}=\left(B_{K}\right)_{l} \bar{K}$. Thus $\left(B_{K}\right)_{l} \bar{K} \cong \operatorname{Hom}_{Z}\left(B_{K}, B_{K}\right)$. This completes the proof of part (2). Thus $B_{K}$ is a central weakly Galois algebra with Galois group $\left.K\right|_{B_{K}} \cong \bar{K}$.

Recall that an algebra $A$ is called an Azumaya weakly Galois extension of $A^{K}$ with Galois group $K$ if $A$ is a weakly Galois extension of $A^{K}$ which is a $C^{K}$-Azumaya algebra where $C$ is the center of $A$. Next, we show that $B_{K} B^{K}$ is an Azumaya weakly Galois extension with Galois group $\left.K\right|_{B_{K} B^{K}} \cong \bar{K}$. We begin with the following two lemmas about $B_{K}$.

LEMMA 3.5. The fixed ring of $B$ under $K, B^{K}=V_{B}\left(B_{K}\right)$.

Proof. For any $b \in B^{K}$ and $x \in J_{g}$ for any $g \in K$, we have that $x b=g(b) x=b x$, so $b \in V_{B}\left(J_{g}\right)$ for any $g \in K$. Thus $b \in V_{B}\left(B_{K}\right)$. Conversely, for any $b \in V_{B}\left(B_{K}\right)$ and $g \in K$, we have that $b x=x b=g(b) x$ for any $x \in J_{g}$, so $(g(b)-b) x=0$ for any $x \in J_{g}$. Hence $(g(b)-b) J_{g}=\{0\}$. But $B J_{g}=J_{g} B=B$ for any $g \in K$, so $(g(b)-b) B=\{0\}$. Thus $g(b)=b$ for any $g \in K$; and so $b \in B^{K}$. Therefore $B^{K}=V_{B}\left(B_{K}\right)$.

LEMма 3.6. The algebra $B^{K}$ is an Azumaya algebra over $Z$ where $Z$ is the center of $B_{K}$.

Proof. Since $B$ is a Galois algebra over $R$ with Galois group $G, B$ is an Azumaya algebra over its center $C$. By the proof of Theorem 3.1, $B_{K}$ is a $C$-separable subalgebra of $B$, so $V_{B}\left(B_{K}\right)$ is a $C$-separable subalgebra of $B$ and $V_{B}\left(V_{B}\left(B_{K}\right)\right)=B_{K}$ by the commutator theorem for Azumaya algebras [3, Theorem 4.3, page 57]. This implies that $B_{K}$ and $V_{B}\left(B_{K}\right)$ have the same center $Z$. Thus $V_{B}\left(B_{K}\right)$ is an Azumaya algebra over $Z$. But, by Lemma $3.5, B^{K}=V_{B}\left(B_{K}\right)$, so $B^{K}$ is an Azumaya algebra over $Z$.

THEOREM 3.7. Let $A=B_{K} B^{K}$. Then $A$ is an Azumaya weakly Galois extension with Galois group $\left.K\right|_{A} \cong \bar{K}$.

Proof. Since $B_{K}$ is a central weakly Galois algebra with Galois group $\left.K\right|_{B_{K}} \cong \bar{K}$ by Theorem $3.4, B_{K}$ is a finitely generated projective module over $Z$ and $\left(B_{K}\right)_{l} \bar{K} \cong$ $\operatorname{Hom}_{Z}\left(B_{K}, B_{K}\right)$. By Lemma 3.6, $B^{K}$ is an Azumaya algebra over $Z$, so $A\left(\cong B_{K} \otimes_{Z} B^{K}\right)$ is a finitely generated projective module over $B^{K}\left(=A^{\bar{K}}\right)$. Moreover, since $B^{K}=V_{B}\left(B_{K}\right)$ by Lemma 3.5 and $\left(B_{K}\right)_{l} \bar{K} \cong \operatorname{Hom}_{Z}\left(B_{K}, B_{K}\right)$,

$$
\begin{aligned}
A_{l} \bar{K} & =\left(B_{K} B^{K}\right)_{l} \bar{K}=\left(B_{K}\right)_{l} \bar{K}\left(B^{K}\right)_{r} \cong B_{K} \bar{K} \otimes_{Z} B^{K} \cong \operatorname{Hom}_{Z}\left(B_{K}, B_{K}\right) \otimes_{Z} B^{K} \\
& \cong \operatorname{Hom}_{B^{K}}\left(B_{K} \otimes_{Z} B^{K}, B_{K} \otimes_{Z} B^{K}\right) \cong \operatorname{Hom}_{B^{K}}\left(B_{K} B^{K}, B_{K} B^{K}\right) \\
& =\operatorname{Hom}_{A^{\bar{K}}}(A, A) .
\end{aligned}
$$


Thus $A$ is a weakly Galois extension of $A^{K}$ with Galois group $\left.K\right|_{A} \cong \bar{K}$. Next, we claim that $A$ has center $Z$ and $A^{\bar{K}}$ is an Azumaya algebra over $Z^{\bar{K}}$. In fact, $B_{K}$ and $B^{K}$ are Azumaya algebras over $Z$ by Theorem 3.1 and Lemma 3.6, respectively, so $A\left(=B_{K} B^{K}\right)$ has center $Z$ and $A^{\bar{K}}=\left(B_{K} B^{K}\right)^{\bar{K}}=B^{K}$. Noting that $B^{K}$ is an Azumaya algebra over $Z$, we conclude that $A^{\bar{K}}$ is an Azumaya algebra over $Z^{\bar{K}}$. Thus $A$ is an Azumaya weakly Galois extension with Galois group $\left.K\right|_{A} \cong \bar{K}$.

4. An Azumaya Galois extension. In this section, we give several characterizations of an Azumaya Galois extension $B$ by using $B_{K}$. This generalizes the results in $[2,6]$. The Z-module $\left\{b \in B_{K} \mid b x=g(x) b\right.$ for all $\left.x \in B_{K}\right\}$ is denoted by $J_{\bar{g}}^{\left(B_{K}\right)}$ for $\bar{g} \in \bar{K}$ where $\bar{K}(=K / L)$ is defined in Lemma 3.2.

LEMMA 4.1. The algebra $B_{K}$ is a central Galois algebra with Galois group $\left.K\right|_{B_{K}} \cong \bar{K}$ if and only if $J_{\bar{g}}^{\left(B_{K}\right)}=\oplus \sum_{l \in L} J_{g l}$ for each $\bar{g} \in \bar{K}$.

Proof. Let $B_{K}$ be a central Galois algebra with Galois group $\left.K\right|_{B_{K}} \cong \bar{K}$. Then $B_{K}=$ $\oplus \sum_{\bar{g} \in \bar{K}} J_{\bar{g}}^{\left(B_{K}\right)}\left[6\right.$, Theorem 1]. Next it is easy to check that $\oplus \sum_{l \in L} J_{g l} \subset J_{\bar{g}}^{\left(B_{K}\right)}$. But $B_{K}=\oplus \sum_{g \in K} J_{g}$, so $\oplus \sum_{g \in K} J_{g}=\oplus \sum_{\bar{g} \in \bar{K}} J_{\bar{g}}^{\left(B_{K}\right)}$ where $\oplus \sum_{l \in L} J_{\mathfrak{g} l} \subset J_{\bar{g}}^{\left(B_{K}\right)}$. Thus $J_{\bar{g}}^{\left(B_{K}\right)}=$ $\oplus \sum_{l \in L} J_{g l}$ for each $\bar{g} \in \bar{K}$. Conversely, since $J_{\bar{g}}^{\left(B_{K}\right)}=\oplus \sum_{l \in L} J_{g l}$ for each $\bar{g} \in \bar{K}$, $B_{K}=\oplus \sum_{g \in K} J_{g}=\oplus \sum_{\bar{g} \in \bar{K}} J_{\bar{g}}^{\left(B_{K}\right)}$. Moreover, by Lemma 3.3, $\left(B_{K}\right)^{K}=Z$, so $\bar{K}$ is a $Z$-automorphism group of $B_{K}$. Hence $J_{\bar{g}}^{\left(B_{K}\right)} J_{\bar{g}^{-1}}^{\left(B_{K}\right)}=Z$ for each $\bar{g} \in \bar{K}$. Thus $B_{K}$ is a central Galois algebra with Galois group $\left.K\right|_{B_{K}} \cong \bar{K}$ because $B_{K}$ is an Azumaya $Z$-algebra by Theorem 3.1 (see [4, Theorem 1]).

Next, we characterize an Azumaya Galois extension $B$ with Galois group $K$.

THEOREM 4.2. The following statements are equivalent:

(1) B is an Azumaya Galois extension with Galois group K;

(2) $Z=C$;

(3) $B=B_{K} B^{K}$;

(4) $B_{K}$ is a central Galois algebra over $C$ with Galois group $\left.K\right|_{B_{K}} \cong K$.

Proof. $(1) \Rightarrow(2)$. Since $B$ is an Azumaya Galois extension with Galois group $K, B^{K}$ is a $C^{K}$-Azumaya algebra. But, by Lemma 3.6, $B^{K}$ is an Azumaya algebra over $Z$, so $Z=C^{K}$. Hence $C \subset Z=C^{K} \subset C$. Thus $Z=C$.

(2) $\Rightarrow(3)$. Suppose that $Z=C$. Then, by Theorem 3.1, $B_{K}$ is an Azumaya algebra over $C$. Hence by the commutator theorem for Azumaya algebras, $B=B_{K} V_{B}\left(B_{K}\right)$ [3, Theorem 4.3, page 57]. But, by Lemma 3.6, $B^{K}=V_{B}\left(B_{K}\right)$, so $B=B_{K} B^{K}$.

(3) $\Rightarrow(4)$. By hypothesis, $B=B_{K} B^{K}$, so $L=\{1\}$ where $L$ is given in Lemma 3.2. By the proofs of Theorem 3.1 and Lemma 3.6, $B_{K}$ and $B^{K}$ are $C$-separable subalgebras of the Azumaya $C$-algebra $B$ such that $B=B_{K} B^{K}$, so $B_{K}$ and $B^{K}$ are Azumaya algebras over $C$ [3, Theorem 4.4, page 58]. Thus $C$ is the center of $B_{K}$. Next, we claim that $J_{g}=J_{g}^{\left(B_{K}\right)}$ for each $g \in K$. In fact, it is clear that $J_{g} \subset J_{g}^{\left(B_{K}\right)}$. Conversely, for each $a \in J_{g}^{\left(B_{K}\right)}$ and $x \in B$ such that $x=y z$ for some $y \in B_{K}$ and $z \in B^{K}$, noting that $B^{K}=V_{B}\left(B_{K}\right)$, we have that $a x=a y z=g(y) a z=g(y) z a=g(y z) a=g(x) a$. Thus $J_{g}^{\left(B_{K}\right)} \subset J_{g}$. This proves that $J_{\mathfrak{g}}=J_{\mathscr{g}}^{\left(B_{K}\right)}\left(=J_{\bar{g}}^{\left(B_{K}\right)}\right.$ since $\left.L=\{1\}\right)$ for each $g \in K$. Hence, $B_{K}$ is a central Galois algebra over $C$ with Galois group $\left.K\right|_{B_{K}} \cong K$ by Lemma 4.1 . 
$(4) \Rightarrow(1)$. Since $B$ is a Galois algebra with Galois group $G, B$ is a Galois extension with Galois group $K$. By hypothesis, $B_{K}$ is a central Galois algebra over $C$ with Galois group $\left.K\right|_{B_{K}} \cong K$, so the center of $B_{K}$ is $C$, that is, $Z=C$. Hence $B^{K}$ is an Azumaya algebra over $C\left(=C^{K}\right)$ by Lemma 3.6. Thus $B$ is an Azumaya Galois extension with Galois group $K$.

Theorem 4.2 generalizes the following result of Kanzaki [6, Proposition 3].

COROLLARY 4.3. If $J_{g}=\{0\}$ for each $g \notin K$, then $B$ is a central Galois algebra with Galois group $K$ and $C$ is a Galois algebra with Galois group $G / K$.

PRoof. This is the case in Theorem 4.2 that $B=B_{K} B^{K}=B_{K}$ where $B^{K}=C$.

We conclude the present paper with two examples, one to illustrate the result in Theorem 4.2, and another to show that $Z \neq C$.

EXAMPLE 4.4. Let $A=\mathbb{R}[i, j, k]$, the real quaternion algebra over the field of real numbers $\mathbb{R}, B=\left(A \otimes_{\mathbb{R}} A\right) \oplus A \oplus A \oplus A \oplus A$, and $G$ the group generated by the elements in $\left\{g_{1}, k_{i}, k_{j}, k_{k}, h_{i}, h_{j}, h_{k}\right\}$ where $g_{1}$ is the identity of $G$ and for all $\left(a \otimes b, a_{1}, a_{2}, a_{3}, a_{4}\right) \in$ $B$,

$$
\begin{aligned}
& k_{i}\left(a \otimes b, a_{1}, a_{2}, a_{3}, a_{4}\right)=\left(i a i^{-1} \otimes b, i a_{1} i^{-1}, i a_{2} i^{-1}, i a_{3} i^{-1}, i a_{4} i^{-1}\right), \\
& k_{j}\left(a \otimes b, a_{1}, a_{2}, a_{3}, a_{4}\right)=\left(j a j^{-1} \otimes b, j a_{1} j^{-1}, j a_{2} j^{-1}, j a_{3} j^{-1}, j a_{4} j^{-1}\right), \\
& k_{k}\left(a \otimes b, a_{1}, a_{2}, a_{3}, a_{4}\right)=\left(k a k^{-1} \otimes b, k a_{1} k^{-1}, k a_{2} k^{-1}, k a_{3} k^{-1}, k a_{4} k^{-1}\right), \\
& h_{i}\left(a \otimes b, a_{1}, a_{2}, a_{3}, a_{4}\right)=\left(a \otimes i b i^{-1}, a_{2}, a_{1}, a_{4}, a_{3}\right), \\
& h_{j}\left(a \otimes b, a_{1}, a_{2}, a_{3}, a_{4}\right)=\left(a \otimes j b j^{-1}, a_{3}, a_{4}, a_{1}, a_{2}\right), \\
& h_{k}\left(a \otimes b, a_{1}, a_{2}, a_{3}, a_{4}\right)=\left(a \otimes k b k^{-1}, a_{4}, a_{3}, a_{2}, a_{1}\right) .
\end{aligned}
$$

Then,

(1) we can check that $B$ is a Galois algebra over $B^{G}$ with Galois group $G$ where $B^{G}=\left\{\left(r_{1} \otimes r_{2}, r, r, r, r\right) \mid r_{1}, r_{2}, r \in \mathbb{R}\right\} \subset C$, and $C=(\mathbb{R} \otimes \mathbb{R}) \oplus \mathbb{R} \oplus \mathbb{R} \oplus \mathbb{R} \oplus \mathbb{R}$, the center of $B$;

(2) $K=\{g \in G \mid g(c)=c$ for all $c \in C\}=\left\{g_{1}, k_{i}, k_{j}, k_{k}\right\}$;

(3) $J_{1}=C, J_{k_{i}}=(\mathbb{R} i \otimes 1) \oplus \mathbb{R} i \oplus \mathbb{R} i \oplus \mathbb{R} i \oplus \mathbb{R} i, J_{k_{j}}=(\mathbb{R} j \otimes 1) \oplus \mathbb{R} j \oplus \mathbb{R} j \oplus \mathbb{R} i \oplus \mathbb{R} j$, $J_{k_{k}}=(\mathbb{R} k \otimes 1) \oplus \mathbb{R} k \oplus \mathbb{R} k \oplus \mathbb{R} i \oplus \mathbb{R} k$, so $B_{K}=\left(A \otimes_{\mathbb{R}} \mathbb{R}\right) \oplus A \oplus A \oplus A \oplus A$. Hence $B_{K}$ has center $C$, that is $Z=C$, and $B_{K}$ is a central Galois algebra over $C$ with Galois group $\left.K\right|_{B_{K}} \cong K$;

(4) $B^{K}=(\mathbb{R} \otimes A) \oplus \mathbb{R} \oplus \mathbb{R} \oplus \mathbb{R} \oplus \mathbb{R}$ and $B=B_{K} B^{K}$, that is, $B$ is an Azumaya Galois extension with Galois group $K$.

EXAMPLE 4.5. Let $A=\mathbb{R}[i, j, k]$, the real quaternion algebra over the field of real numbers $\mathbb{R}, B=A \oplus A \oplus A, G=\left\{1, g_{i}, g_{j}, g_{k}\right\}$, and for all $\left(a_{1}, a_{2}, a_{3}\right) \in B$,

$$
\begin{aligned}
& g_{i}\left(a_{1}, a_{2}, a_{3}\right)=\left(i a_{1} i^{-1}, i a_{2} i^{-1}, i a_{3} i^{-1}\right), \\
& g_{j}\left(a_{1}, a_{2}, a_{3}\right)=\left(j a_{1} j^{-1}, j a_{3} j^{-1}, j a_{2} j^{-1}\right), \\
& g_{k}\left(a_{1}, a_{2}, a_{3}\right)=\left(k a_{1} k^{-1}, k a_{3} k^{-1}, k a_{2} k^{-1}\right) .
\end{aligned}
$$


Then,

(1) $B$ is a Galois algebra over $B^{G}$ where $B^{G}=\left\{\left(r_{1}, r, r\right) \mid r_{1}, r \in \mathbb{R}\right\} \subset C$, and $C=$ $\mathbb{R} \oplus \mathbb{R} \oplus \mathbb{R}$, the center of $B$. The $G$-Galois system is $\left\{a_{i} ; b_{i} \mid i=1,2, \ldots, 8\right\}$ where

$$
\begin{aligned}
& a_{1}=(1,0,0), \quad a_{2}=(i, 0,0), \quad a_{3}=(j, 0,0), \quad a_{4}=(k, 0,0), \\
& a_{5}=(0,1,0), \quad a_{6}=(0, j, 0), \quad a_{7}=(0,0,1), \quad a_{8}=(0,0, k) \text {; } \\
& b_{1}=\frac{1}{4} a_{1}, \quad b_{2}=-\frac{1}{4} a_{2}, \quad b_{3}=-\frac{1}{4} a_{3}, \quad b_{4}=-\frac{1}{4} a_{4} \text {, } \\
& b_{5}=\frac{1}{2} a_{5}, \quad b_{6}=-\frac{1}{2} a_{6}, \quad b_{7}=\frac{1}{2} a_{7}, \quad b_{8}=-\frac{1}{2} a_{8},
\end{aligned}
$$

(2) $K=\{g \in G \mid g(c)=c$ for all $c \in C\}=\left\{1, g_{i}\right\}$ where $J_{g_{i}}=\mathbb{R} i \oplus \mathbb{R} i \oplus \mathbb{R} i$, so $B_{K}=$ $\mathbb{R}[i] \oplus \mathbb{R}[i] \oplus \mathbb{R}[i]$ which is a commutative ring not equal to $C$, that is, $Z \neq C$.

ACKNOWLEDGMENTS. This work was supported by a Caterpillar Fellowship at Bradley University. The authors would like to thank the Caterpillar Inc. for the support.

\section{REFERENCES}

[1] R. Alfaro and G. Szeto, On Galois extensions of an Azumaya algebra, Comm. Algebra 25 (1997), no. 6, 1873-1882.

[2] F. R. DeMeyer, Galois theory in separable algebras over commutative rings, Illinois J. Math. 10 (1966), 287-295.

[3] F. R. DeMeyer and E. Ingraham, Separable Algebras over Commutative Rings, Lecture Notes in Mathematics, vol. 181, Springer-Verlag, Berlin, 1971.

[4] M. Harada, Supplementary results on Galois extension, Osaka J. Math. 2 (1965), 343-350.

[5] _ Note on Galois extension over the center, Rev. Un. Mat. Argentina 24 (1968/1969), no. 2, 91-96.

[6] T. Kanzaki, On Galois algebra over a commutative ring, Osaka J. Math. 2 (1965), 309-317.

[7] G. Szeto and L. Xue, The structure of Galois algebras, J. Algebra 237 (2001), no. 1, 238-246.

[8] O. E. Villamayor and D. Zelinsky, Galois theory with infinitely many idempotents, Nagoya Math. J. 35 (1969), 83-98.

George SZeto: Department of Mathematics, Bradley University, Peoria, IL 61625, USA E-mail address: szeto@hi11 top.brad1ey.edu

Lianyong Xue: DePartment of Mathematics, Bradley University, PeORIA, IL 61625, USA E-mail address: 1xue@hi 17top.brad7ey.edu 


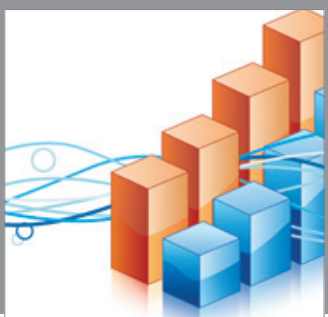

Advances in

Operations Research

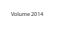

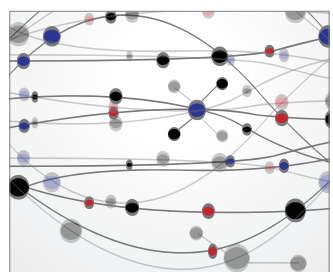

\section{The Scientific} World Journal
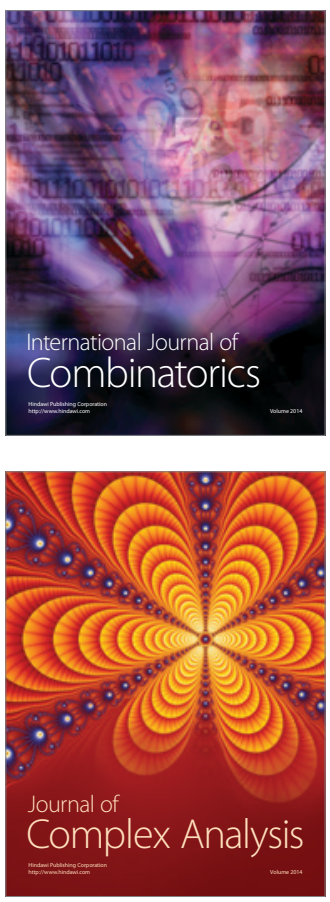

International Journal of

Mathematics and

Mathematical

Sciences
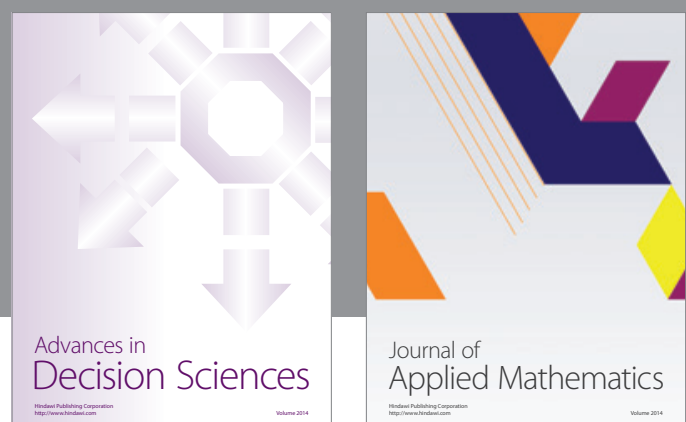

Journal of

Applied Mathematics
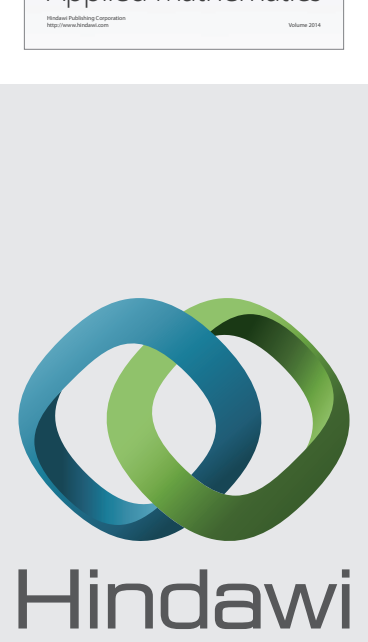

Submit your manuscripts at http://www.hindawi.com
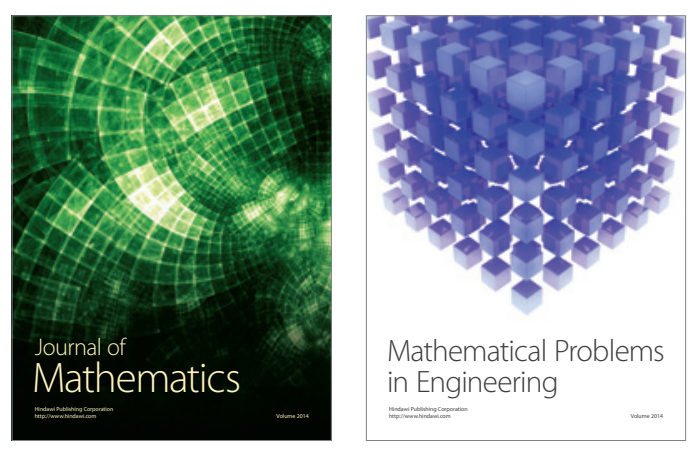

Mathematical Problems in Engineering
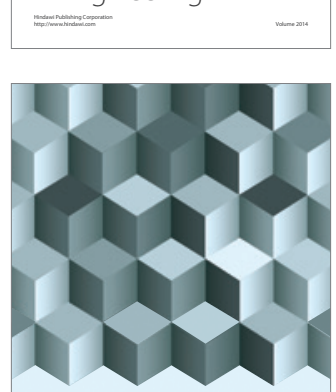

Journal of

Function Spaces
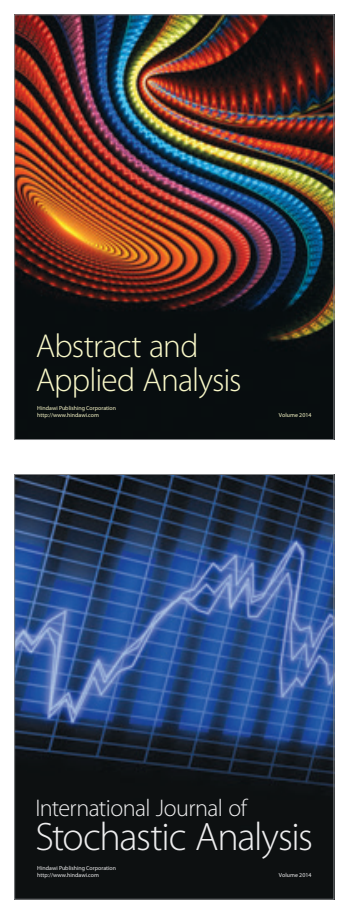

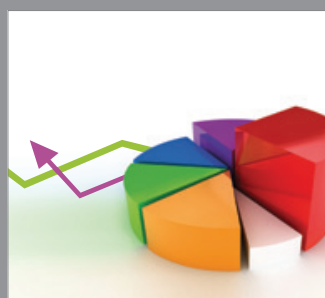

ournal of

Probability and Statistics

Promensencen
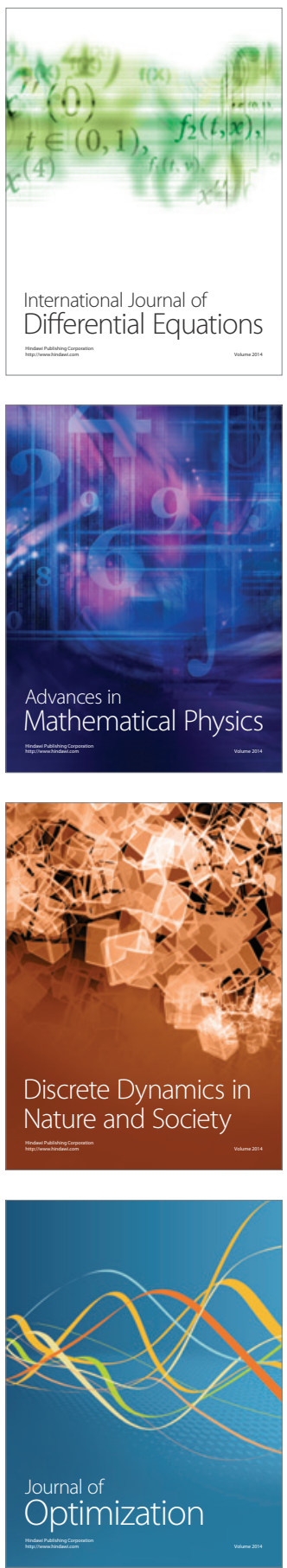